der CDU eröffnete, seit dem Bruch der Koalition von der FDP unterstützte Trommelfeuer gegen die Universität beendet. Mit der Bildung der sozialdemokratischen Alleinregierung begann eine - bis jetzt noch nicht voll einschätzbare - neue Phase der Beziehungen zwischen Universität und Land. Immerhin brachte die Regierungserklärung vom I 5. I2. I971 die vor der Wahl von der FDP mühsam, von der SPD behutsam offengehaltene - Option für die Einführung und Erprobung der einstufigen Juristenausbildung in Bremen. Auf erneute Bitte der Universität beschloß unter Vorsitz des neuen Senators für Rechtspflege und Strafvollzug die Fachdeputation am 29. I 2. 197 I die halbparitätische Beteiligung der Universität (einschließlich des Referendarvertreters) an der Sachverständigenkommission (unter Vergrößerung auf 22 Mitglieder). Der fortbestehende Unterausschuß präzisierte den Auftrag der Kommission am 17. I 2. 1972 dahingehend, daß sie „... bis Ende Mai dieses Jahres den Entwurf eines Juristenausbildungsgesetzes für eine einstufige Juristenausbildung und den Abriß eines Ausbildungsmodells ... (vorlegen solle). Mit dem Gesetz soll ein einjähriges integriertes sozialwissenschaftliches Eingangsstudium erprobt werden ... (Weser-Kurier, I 8. I. 1972, I4).

Die Kommission nahm ihre Arbeit am 17. I. 1972 mit der Bildung von 4 Arbeitsgruppen (Sozialwissenschaftliches Eingangsstudium; Juristischer Stoffplan; Verbindung von Theorie und Praxis; Studienbegleitende Kontrollen, Abschlußverfahren, Ausbildungs- und Prüfungsamt) und der Vereinbarung eines weiteren Hearings über $»$ Projektstudium in der Juristenausbildung " wieder auf.

2.I 3 Am 14. I. 1972 hatte sich die - bis auf 2 Vertreter der PKS - aus Vertretern der Hochschullehrer und Studenten der Universität Bremen gebildete Studiengangskommission Rechtswissenschaften konstituiert; sie arbeitet mit den Vertretern der Universität in der Landeskommission engstens zusammen.

In Anlehnung an die - schon erwarteten - Themengruppen der Landeskommissionsarbeit hat die Studiengangskommission eigene Arbeitsgruppen gebildet. Diese entwickeln zur Zeit - koordiniert durch das Plenum - ein Rahmen-curriculum für eine projektorientierte Ausbildung für Juristen im Rahmen der geplanten - ebenfalls projektbezogenen - Studienbereichsstruktur der Universität, das der im bremJAG zu konkretisierenden Experimentierklausel des DRiG genügt.

\title{
Das Bundesausbildungsförderungsgesetz: Förderung der Ausbildung oder der Ausbeutung?
}

Der Ausbildungssektor befindet sich heute in einer Krise, wer wüßte das nicht. Selbst der illustrierte Blätterwald bejammert die mangelnde Repräsentation der unteren Klassen an Hochschulen, höheren Schulen und ähnlichen Bildungseinrichtungen. Die Stunde der Reformen schlägt, es müssen Gesetze geschaffen werden, die die gesellschaftliche Misere beseitigen, sonst beseitigt etwa noch die gesell- 
schaftliche Misere die Gesellschaft. Eines dieser vielen Gesetze ist das BAföG vom 19. 9. 1969 und jetzt in einer neuen Fassung vom 24. 6. 1971. Es trägt das Fluidum allen sozialdemokratischen Reformeifers: Verwirklichung des Sozialstaates, Leistungen des Staates unter Führung der Sozialdemokratie für die armen Menschen, garniert mit suggestiven Schlagwortparaden aus der liberalen Mottenkiste, eben zum besseren Verständnis für die armen Menschen. "Das Ausbildungsförderungsgesetz stellt einen entscheidenden Schritt zur Verwirklichung gleicher Chancen in der Ausbildung dar «, erklärt uns die Bundesministerin für Jugend, Familie und Gesundheit, Käte Strobel. Im BAföG dreht es sich nur um Geld, das in einer Kette von Paragraphen in Höchstbeträgen unter bestimmten Voraussetzungen in Prozenten berechnet und verteilt wird. Der in minutiöse Abschnitte und Unterabschnitte gegossene Geldfetisch soll unsere Gesellschaftsordnung demokratisieren, soll zumindest die Krise im Ausbildungssektor beseitigen helfen, denn Geld regiert die Welt. Daß aber klassenbedingte sozialpsychologische Hemmnisse viel eher der Grund dafür sind, daß die Kinder des Fließbandarbeiters nicht zur höheren Schule gehen, kann unter diesem Blidkwinkel natürlich niemals zum Problem werden; ganz im Gegenteil, solches wird fein säuberlich aus dem BAföG heraussortiert und der Verantwortung sogenannter struktureller Bildungsreformen unterstellt. Wann diese Reformen und vor allem wie diese kommen werden, soll hier nicht Gegenstand unserer Sorge sein. Betrachten wir jetzt aber näher, was unser gefeierter Sozialstaat im BAföG zu bieten hat.

\section{Der Bereich der Förderung}

Die Leistungen nach dem BAföG kann nur in Anspruch nehmen, wer eine der folgenden Ausbildungsstätten besucht: Gymnasien und Fachoberschulen ab Klasse II - Abendhauptschulen - Berufsaufbauschulen - Abendrealschulen Abendgymnasien und Kollegs - Berufsfachschulen, soweit für deren Besuch der Realschulabschluß oder eine vergleichbare Vorbildung Voraussetzung ist Fachschulen - höhere Fachschulen - Akademien und Hochschulen - Fernunterrichtslehrgänge und Praktika, die einen der oben genannten Bildungsabschlüsse vorbereiten. Bei der Beratung des Gesetzes war geplant, daß auch die einfachen Berufsschulen in den Förderungsbereich fallen sollten. Der finanzielle Kostenvoranschlag ergab jedoch, daß der Sozialstaat für Lehrlinge leider nicht die notwendigen Ressourcen aufbringen kann, denn ca. $70 \%$ der Jugendlichen sind heute Lehrlinge. Lehrlinge müssen sich weiterhin mit Unterstützungsbeiträgen nach dem Arbeitsförderungsgesetz begnügen. Es ist zwar zu beobachten, daß die Lehrlingsgehälter in den letzten Jahren stellenweise angestiegen sind, Monatsbeträge von ca. $270 \mathrm{DM}$ stellen aber durchaus keine Seltenheit dar. Dabei wird die Arbeitskraft des Lehrlings meist weit über den Bereich hinaus in Anspruch genommen, der mit der Ausbildung zu tun hat. Käte Strobels Chancengleichheit ist also offensichtlich für das Gros der Jugendlichen nichts anderes als Geschwätz. Der Lehrling nämlich sieht sich in der Berufsschule mit einer Realität konfrontiert, die mit Ausbildung zu einem bestimmten Beruf, den er technisch und gesellschaftlich beherrschen muß, wohl kaum noch etwas zu tun hat. Die Logik des kapitalistischen Produktionsprozesses erfordert Arbeitskräfte, denen der Umgang mit dem Maschinensystem spielend gelingt, damit sie Mehrwert produzieren können, nicht Arbeiter, die den Produktionsprozeß nach der Logik gesellschaftlicher Bedürfnisse gestalten wollen. 
Wer allerdings meint, daß die Ausbildungsbereiche, die unter das BAföG fallen, nicht so rigide der Logik des Kapitals gehorchen, soll sich getäuscht sehen. Das BAföG knüpft die Förderungsdauer z. B. an der Hochschule an das sog. Regelstudium. Dieses Regelstudium wird nach dem geplanten Hochschulrahmengesetz hauptsächlich das 6 Semester umfassende Kurzstudium sein. Was ein Student, der auf die Förderung angewiesen ist, sich in diesen 6 Semestern aneignen kann, ist höchstens ein komprimierter Stoff, den er bei einer Prüfung maschinenmäßig abspulen kann. Wer allerdings das BAföG nicht benötigt, weil er genügend Geld zur Verfügung hat, kann sich da etwas mehr Zeit lassen. Gerade an diesem Punkt mag man sich sagen, also ist doch die Bereitstellung finanzieller Mittel sehr vorteilhaft, braucht doch dann derjenige, der nicht die erforderlichen finanzkräftigen Eltern hat, sein Studium nicht mehr durch Werksarbeit oder ähnliches zu finanzieren. Also doch eine erfreuliche Leistung unseres Sozialstaates?! Wie läßt sich diese menschenfreundliche Haltung des Staates, die in der Tat ein Beitrag zur Chancengleichheit zu sein scheint, erklären? Lassen wir die Bundesregierung sprechen; In den kommenden Jahrzehnten werden in Wissenschaft, Wirtschaft und Bildungswesen unseres Landes die benötigten Mitarbeiter nur zur Verfügung stehen, wenn es gelingt, die "Bildungsreserven « $z u$ »aktivieren«. Die liberale Chancengleichheit ist hier schon zum schlichten technischen Expressionismus geronnen. Jovialer und einladender ist da schon die Sprache des Unternehmerbriefes des Deutschen Industrie Instituts: »Die Wirtschaft wird es daher begrüßen, schon nach drei Jahren berufstätige junge Menschen in die Unternehmen zu bekommen. "Hier kann man exemplarisch erkennen, was hinter der demokratisch und sozial klingenden Verpackung der Chancengleichheit als Inhalt stedkt: das Bedürfnis des Kapitals nach Arbeitskräften mit einer Qualifikationsstruktur, die dem technologisch gewandelten Produktionsprozeß gerecht wird. Das Chancengleichheitspostulat läßt sich auf ökonomische Effizienz reduzieren. »Gleiche Bildungschancen werden solange gewährt, bis Aufwand und Ertrag der Gewährung von Bildungschancen eine bestimmte Normgröße nicht übersteigt. « ${ }^{1}$ Liberalsoziale Ideologie in Raten, Chancengleichheit nur so lange, bis die Bedürfnisse des Kapitals gesättigt sind. Damit aber niemand das Chancengleichheitspostulat zu ernst nimmt, hat sich die Bildungsökonomie neue Zauberworte einfallen lassen: "Reichtum", » wachsender Wohlstand «, Überschuß an nachgefragten Arbeitskräften « etc. Erst in dem Augenblick, wo Reichtum und wachsender Wohlstand erreicht seien, könne man überhaupt die »individuellen "Wünsche nach »echter * Bildung verwirklichen. $\mathrm{Da}$ man heute freilich Reichtum nicht als stofflichen Reichtum versteht, sondern Reichtum an Mehrwert, spricht man vom St. Nimmerleinstag, denn wann wird es im Kapitalismus genügend Profit geben?? Diese Vertröstung auf später hat ideologische Alibifunktionen. ${ }^{2}$ Wer allerdings sich nicht so einfach der Bildungsstrategie des Kapitalismus unterordnen will und beispielsweise in Streiks auch nur fordert, daß Bildung andere Bezugspunkte haben könnte als die Profitinteressen des Kapitals, sieht sich mit dem Paragraphen 20 des geplanten Hochschulrahmengesetzes konfrontiert. Dieser bestimmt im Zusammenhang mit dem BAföG, daß die Studien-

1 H. P. Widmeier, Bildung u. Wirtschaftswachstum, Villingen r 966 S. 30.

2 F. Huisken, Kurzdarstellung marxistischer Kategorien, die zur Kritik der bürgerlichen Bildungsökonomie diencn können, in: Altvater Huisken, Materialien zur politischen Okonomie des Ausbildungssektors, Erlangen I97r, S. 192. 
förderung dann wegfällt, »wenn der Auszubildende die Ausbildung aus einem von $\mathrm{ihm}$ zu vertretenden Grund unterbrochen hat.*

\section{Die Höbe der Förderungsbeträge und die Voraussetzungen der Leistungen}

Das BAföG zeigt nicht nur die Abhängigkeit des Sozialstaates von den Bedürfnissen des Kapitals, sondern auch sein knauseriges Sozialverständnis. Der höchste Betrag, der überhaupt ausgezahlt werden kann, ist $420 \mathrm{DM}$; dieser wird gezahlt für Besucher von höheren Fachschulen, Akademien und Hochschulen, sofern der Lernende außerhalb des elterlichen Hauses lebt. Davon soll man wohnen, essen, sich kleiden und lesen können. Zwar errechnete das Deutsche Studentenwerk bereits einen Minimalbetrag von 520 DM für Studenten, aber der Sozialstaat scheint da mehr von den Worten des feudalen Handwerkermeisters zu halten: "Lehrjahre sind keine Herrenjahre." Die Höhe der Leistungen hängt ab von der Investitionsbereitschaft des Sozialstaats in den Ausbildungssektor. $Z_{\text {war soll }}$ die »reformierte « Ausbildungsstruktur die Voraussetzungen der Profitproduktion verbessern, aber sie kann nicht direkt Profit bringen. Die Unsicherheit darüber, ob die vermehrten Bildungsausgaben wirklich auch vermehrte Profite bedingen, findet ihren Ausdruck darin, daß die Ausbildungskosten möglichst gering gehalten werden müssen, wobei die Effektivität genauestens zu kalkulieren ist. Allzu viel Ausgaben könnten, so ängstigen sich die Bildungsplaner, eine "Überqualifikation « der Arbeitskraft mit sich bringen. Damit aber eventuell auftretende Effektivitätslücken nicht unnötig die Profitinteressen und -erwartungen mit Unsicherheit belasten, werden vermehrte Bildungsausgaben hauptsächlich nicht etwa durch Profitabzüge finanziert, sondern durch Lohnabzüge in Form der Lohnsteuer.

Auch auf soziale Rangfolgen legt der Sozialstaat größten Wert, im Gegensatz zum Studenten erhält der einfachere Berufsfachschüler nur 320 DM monatlich. Offensichtlich liest der simplere Berufsfachschüler weniger. ${ }^{3}$ Freilich hat das BAföG neben solchen verkümmerten Sozialleistungen auch etwas zu bieten; wer nämlich unter die gesetzlichen Voraussetzungen fällt, hat heute einen Rechtsanspruch auf Förderung. Das muß sein, da wir heute auch in einem sogenannten Rechtsstaat leben. Jeder Auszubildende hat das Recht, eine Bildung zu erwerben, die die Profitinteressen des Kapitals befriedigt, und er hat das Recht und die Freiheit, Bildung sich unter den miserabelsten Bedingungen anzueignen.

Natürlich erhält nicht jeder, der die obengenannten Ausbildungsbereiche besucht, diesen Betrag zur Verfügung gestellt. Ob man sozialstaatliche Unterstützung erhält oder nicht, hängt ab vom Einkommen der Eltern, obwohl Ministerin Strobel im Zusammenhang mit dem BAföG erzählt, daß es wesentlich darauf ankomme, wallen jungen Menschen die gleichen Start- und Ausbildungschancen unabhängig vom Einkommen der Eltern zu sichern.« Tatsache ist, daß der volle Förderungsbetrag nur dann gezahlt wird, wenn der Vater nicht mehr als $800 \mathrm{DM}$ netto im Monat verdient; sofern beide Elternteile berufstätig sind, darf das ge-

3 Die Höhe der anderen Förderungsbeträge beträgt in den Bereichen (in der Klammer stehend der Betrag, sofern der Auszubildende zu Hause lebt): Gymnasien, Berufsfachschulen, Fachoberschulen ohne Zugangsvoraussetzung einer abgeschlossenen Berufsausbildung ( $160 \mathrm{DM}) 320 \mathrm{DM}$ - Fachoberschulen mit Zugangsvoraussetzung einer abgeschlossenen Berufsausbildung, Abendhauptschulen, Berufsaufbauschulen, Abendrealschulen (320 DM) 380 DM - Fachschulen, Abendgymnasien und Kollegs (320 DM) 400 DM - Höhere Fadschulen, Akademien und Hodıschulen (340 DM) $420 \mathrm{DM}$. Bei einer jährlichen Inflationsrate von mindestens $4 \%$ stellen diese Beträge wahrlich ein Hungergehalt dar. 
meinsame Nettoeinkommen nicht 930 DM übersteigen. ${ }^{4}$ Hat der zu Fördernde eigenes Einkommen, so darf gem. $\$ 23$ BAföG sein Einkommen nicht höher als I 25 DM monatlich sein, um den vollen Förderungsbetrag zu erhalten. ${ }^{5}$ Auch das Einkommen des verdienenden Ehegatten wird veranschlagt: 500 DM netto monatlich und nicht mehr, sonst gibt es den vollen Förderungsbetrag nicht.

Die Verfasser des Gesetzestextes waren bei der Formulierung der Einkommensgrenzen offensichtlich weniger vom Vorbild der Sozialstaatstheorie geleitet als vielmehr von der Kollekte an den Pforten der Kirche für die Ärmsten der Gemeinde. Aber, aber werden uns die Spezialisten des Sozialstaates entgegnen, man erhält doch auch die Ausbildungsförderung, wenn das Einkommen der Eltern die gesetzlichen Einkommensgrenzen übersteigt. Ja, das stimmt, auch dann kann man noch ein paar Almosen erhalten, mit steigendem Einkommen immer weniger, Beträge unter so DM werden nicht mehr ausgezahlt. Aber die Eltern, die das Unglück haben, zwei Kinder zu besitzen und das noch größere Unglück, $\mathrm{da} ß$ eines dieser Kinder sich gemäß den Bedingungen unseres Sozialstaates ausbilden lassen will, können sich drehen und wenden, addieren, subtrahieren, wie sie wollen, wenn das monatliche Einkommen höher ist als I 370 DM netto, dann gibt es keinen Pfennig Ausbildungshilfe. ${ }^{6}$ Die familienunabhängige Förderung ist also nicht verwirklicht. Falsch ist es aber, hierüber mit dem großen Lamentieren zu beginnen, der Jugendliche befinde sich immer noch in Abhängigkeir von den Eltern. Entscheidend ist vielmehr - ob familienabhängig oder -unabhängig - die Abhängigkeit des Lernenden vom Staat, auf dessen Gnade und Absichten er angewiesen ist. Ein Heer von staatlichen Ämtern für Ausbildungsförderung wacht über die Verwirklichung dieser Absichten: Man soll nur das lernen, was eine bessere Ausbeutung der eignen Arbeitskraft gewährleistet oder ebenso fatal, wie man fremden Arbeitskräften rationaler den Mehrwert auspressen kann.

Henry Düx

\footnotetext{
- Sind die Eltern geschieden oder leben sie dauernd getrennt oder lebt nur noch ein Elternteil, so darf das Einkommen nicht soo DM übersteigen. Diese Einkommensgrenzen erhöhen sich, wenn die Eltern zusätzliche Personen zu versorgen haben, für Unterhaltsberechtigte, die nicht Kinder sind, etwa der Großvater, um $270 \mathrm{DM}$, bei Kindern, die in einer förderungsfähigen Ausbildung stehen um so DM, bei Kindern, die das ig. Lebensjahr noch nidht vollendet haben ummonatlich $200 \mathrm{DM}$, bei Kin* dern die das Is. Lebensjahr vollendet haben um 270 DM. In einer Familie, in der nur der Vater verdient (Einkommensgrenze 800 DM), ein 17jähriger Gymnasiast einen Antrag auf Ausbildungsförderung stellt (Kind, das in förderungsfähiger Ausbildung ist, Erhöhung der Einkommensgrenze um so DM und der eine I Ijährige Scwester hat (wreitere Erhöhung der Grenze um 200 DM), darf das Einkommen des Vaters I050 DM nicht übersteigen, damit der Gymnasiast den vollen Förderungsbetrag erhält.

5 In manchen Bildungsbereichen beträge die Einkommensgrenze sogar nur 75 DM monatlich.

- Für den Fall, daß das Einkommen die Grenzbeträge übersteigt, sind die Einkommensüberschüsse als Eigenverwendung für die Ausbildung anzusehen. Ein Berufsfachschüler, der monatlich roo DM verdient und bei dem die Einkommensgrenze 75 DM beträgt, muß die überschießenden 25 DM für die Ausbildung aufwenden. Der Pauschalsatz, den Berufsfachschüler in Höhe von $320 \mathrm{DM}$ erhalten vermindert sidh dann um $25 \mathrm{DM}$, er erhält nur noch $295 \mathrm{DM}$. Bei dem Einkommen der Eltern ist es etwas komplizierter. Hat im obigen Beispiel (Anm. 4) der Vater ein Einkommen von 1200 DM (errednete Einkommensgrenze roso DM) so übersteigt der Verdienst diese Grenze um I50 DM. Hier bestimmt $\$ 25$ Abs. 4 BAföG, daß nicht die ganzen I so DM auf den Pauschalsatz (bei Schülern des Gymnasiums zu Hause I60 DM angerechnet werden. Das wären dann nur noch so DM Beihilfe. Von den iso DM bleiben für die Eltern $40 \%$ anrechnungsfrei, d. h. müssen nicht für Ausbildung aufgewandt werden, für jedes Kind bleiben weitere $5 \%$ anrechnungsfrei, bei zwei Kindern $10 \%$, also insgesamt $50 \%$. Die Eltern haben also nur $50 \%$ von I 50 DM selbst aufzubringen. Bei dem Pauschalsatz von $160 \mathrm{DM}$ müssen sich die Eltern folglich mit $75 \mathrm{DM}$ beteiligen, sie erhalten vom Staat nur noch 85 DM.
} 
Wer sich über das kürzlich in Kraft getretene neue Betriebsverfassungsgesetz (BVG) ausschließlich durch die Stellungnahme der Unternehmerverbände informieren läßt, wird glauben, mit diesem Gesetz sei in der Bundesrepublik der Sozialismus eingeführt worden. So heißt es, den Unternehmern solle »die Verantwortung für die Unternehmenspolitik aus der Hand geschlagen werden ${ }^{1}$, ihre »notwendige Entscheidungsbefugnis « werde »in wesentlichen Bereichen unmöglich gemacht $\ll^{2}$, ihr Freiheitsraum »zum Teil vollständig ausgeböblt $\aleph^{3}$ usw. $\mathrm{Daß}$ diese Einschätzung offensichtlich falsch ist, liegt auf der Hand. Das neue BVG hat die betriebliche Herrschaftsstruktur nicht wesentlich verändert. Es enthält zwar im einzelnen einige Verbesserungen der Einflußmöglichkeiten des Betriebsrats, bindet diesen aber weiterhin durch die Verpflichtung zu »vertrauensvoller Zusammenarbeit « an das unternehmerische Profitinteresse. Die Klagelieder der Unternehmer sind nur Teil einer Strategie, jede Beschränkung ihrer nAutonomie" - vom Standpunkt der Lohnabhängigen heißt das: der innerbetrieblichen Herrschaft - als Eingriff in »unsere freiheitliche Ordnung ren und somit abzuwehren.

In dieser Abwehrstrategie ist der Rechtswissenschaft eine wichtige Rolle zugedacht: Durch einschränkende Interpretation der Mitbestimmungsrechte soll sie dazu beitragen, diese zu unterlaufen. Wie man das am besten anstellt, haben 16 Professoren des Arbeitsrechts am 6. I I. I971 auf einer "Gesprächsveranstaltung der Bundesvereinigung Deutscher Arbeitgeberverbände « (BDA) diskutiert. Beteiligt waren u. a. die Professoren: Boldt, Bötticher, Adomeit, Hanau, Hueck, Löwisch, Säcker, Lieb, Richardi, Galperin, Kraft, Zöllner, Dütz, Gamillscheg und Rüthers. ${ }^{4}$ Hierbei handelt es sich durchgehend um "renommierte " Arbeitsrechtler. Drei von ihnen sind Mitglieder der Sachverständigenkommission der Bundesregierung für ein neues Arbeitsgesetzbuch (Dütz, Gamillscheg und Rüthers). Diese Kommission tagte ebenfalls am 6. I I. I97I, als die "Gesprächsveranstaltung " der BDA stattfand. Die drei Professoren nahmen die Einladung der Unternehmer wichtiger und schwänzten die Kommissionssitzung. ${ }^{5}$

Ein Teil der Professoren hatte sich schon vor der »Gesprächsveranstaltung * für die ihnen von der BDA zugedachten Aufgaben qualifiziert, indem sie den damaligen Regierungsentwurf für ein neues BVG im Sinne der Unternehmer interpretiert und insbesondere die Verfassungswidrigkeit wesentlicher Bestimmungen behauptet hatten. So vertritt Galperin in einem Rechtsgutachten für die Unternehmerseite ${ }^{8}$ die Ansicht, wichtige Teile des Regierungsentwurfs verstießen gegen das Grundgesetz. Der Entwurf enthalte »eine ganze Reihe von radikalen Eingriffen in die unternehmerische Freiheit « (S. 6). Galperin sieht zum Beispiel in der Erstreckung der erzwingbaren Mitbestimmung auf die Festsetzung sämtlicher Bezugsgrößen von leistungsbezogenen Arbeitsentgelten nach $\$ 87$ Abs. I,

1 H. M. Schleyer, zitiert nach konkret $3 / 1972$, S. 9 .

2 Kritische Anmerkungen zum Regierungsentwurf, hrsg. von der BDA, 1971, S. I.

3 L. Joens, Mitbestimmung - Rückschritt nach links?, hrsg. vom Wirtschaftsrat der CDU e. V., I97 I, S. 20.

4 Nach Das Mitbestimmungsgespräch I/1972, S. 2. Vgl. auch Der Spiegel so/1971, S. 95 .

5 Vgl. Das Mitbestimmungsgespräch ebd. und W. Fritzc (Leirer der DGB-Bundespressestelle) in Der Spiegel 53/1971, S. I I (Leserbrief).

- H. Galperin, Der Regierungsentwurf eines neuen Betriebsverfassungsgesetzes, Düsseldorf I 97 I. 